\title{
A Novel Hysteresis Model of Magnetic Field Strength Determined by Magnetic Induction Intensity for Fe-3\% Si Electrical Steel Applied in Cigarette Making Machines
}

\author{
Hao Wang, ${ }^{1}$ Jianbo Zhan, ${ }^{1}$ Zhenhua Yu, ${ }^{1}$ Ying Zhang, ${ }^{1}$ Jiang Yu, ${ }^{1}$ \\ Yongfa Gui, ${ }^{1}$ Tingting Yu, ${ }^{1}$ Jiao Xie, ${ }^{1}$ Hongkui Zhang, ${ }^{2}$ Yafeng Ji, \\ Na Zan, ${ }^{4}$ Rongrong Fu, ${ }^{5}$ and Deniz Perin ${ }^{6}$ \\ ${ }^{1}$ R\&D Center, China Tobacco Yunnan Industrial Co., Ltd., Kunming, Yunnan 650231, China \\ ${ }^{2}$ Fushun Branch of China Coal Research Institute, Fushun 113001, China \\ ${ }^{3}$ Heavy Industry Engineering Center of China Ministry of Education, Taiyuan University of Science and Technology, \\ Taiyuan, Shanxi 030024, China \\ ${ }^{4}$ AVIC Commercial Aircraft Engine Co., Ltd., Shanghai 200241, China \\ ${ }^{5}$ Measurement Technology and Instrumentation Key Laboratory of Hebei Province, Yanshan University, Qinhuangdao, \\ Hebei 066004, China \\ ${ }^{6}$ Department of Physics, Balıkesir University, 10145 Balkesir, Turkey
}

Correspondence should be addressed to Hao Wang; neuwanghao@gmail.com

Received 2 April 2016; Accepted 27 July 2016

Academic Editor: Mohindar S. Seehra

Copyright (C) 2016 Hao Wang et al. This is an open access article distributed under the Creative Commons Attribution License, which permits unrestricted use, distribution, and reproduction in any medium, provided the original work is properly cited.

Hysteresis characteristics of grain-oriented electrical steel were studied through the hysteresis loop. Existing hysteresis fitting simulation methods were summarized, and new Fe-3\% Si grain-oriented electrical steel hysteresis loop model was proposed. Undetermined coefficients of the magnetic field intensity and magnetic flux density were determined by both the fixed angle method and the least squares method, and the hysteresis loop model was validated with high fitting degree by experimental data.

\section{Introduction}

Fe-3\% Si grain-oriented electrical steel is an important soft magnetic material, which is often used for cigarette machine sensor because of its high magnetic flux density and good magnetic performance [1-5]. Therefore, parameters such as iron loss, permeability, shielding efficiency, and Barkhausen noise of $\mathrm{Fe}-3 \% \mathrm{Si}$ grain-oriented electrical steel have been studied extensively. When the magnetic flux is alternating, hysteresis and eddy current losses will be generated. Existence of hysteresis and eddy current losses will make the magnetic flux density lag behind the magnetic field intensity; after repeatedly magnetizing multiple cycles, the hysteresis loop forms $[6,7]$. Applied sensors in cigarette making machines usually do not consider the complex effects of the hysteresis loop, inevitably leading to errors in the following simulation or performance prediction, which is an important reason why switch protection of cigarette making machine sensor is not accurate enough [8-10].

Hysteresis is a quite important magnetic property of $\mathrm{Fe}-$ $3 \%$ Si grain-oriented electrical steel, since its hysteresis loops and magnetization curves reflect the significant characteristics of the material, which is also an important basis for the selection of cigarette machine sensor materials [11, 12]. Study on the material hysteresis loop model is helpful to better understand the hysteresis property of the material, which is significant to the design of materials and related components [13]. With the increasingly higher demand of equipment design accuracy in the actual operation, it is necessary to accurately determine the impact caused by hysteresis $[14,15]$. In the characterization analysis of motors, transformers, and other electromagnetic equipment, it is the first step to obtain 
TABLE 1: Chemical composition of $\mathrm{CCl}$ and $\mathrm{CH} 1$ tested materials (mass percent, \%).

\begin{tabular}{lcccccccc}
\hline Sample & $\mathrm{Si}$ & $\mathrm{Mn}$ & $\mathrm{C}$ & $\mathrm{Cu}$ & $\mathrm{S}$ & $\mathrm{P}$ & $\mathrm{Al}$ & $\mathrm{Fe}$ \\
\hline $\mathrm{CC} 1$ & 3.05 & 0.065 & 0.032 & 0.028 & 0.020 & 0.012 & 0.008 & Balance \\
$\mathrm{CH} 1$ & 3.09 & 0.072 & 0.054 & 0.075 & 0.018 & 0.015 & 0.010 & Balance \\
\hline
\end{tabular}

accurate waveform relationship of $B-H$ loop [16]. When the electromagnetic fields are accurately calculated, there must be a more accurate and rapid method for determining the $B-H$ curve [17].

$\mathrm{Fe}-3 \% \mathrm{Si}$ grain-oriented electrical steel hysteresis performance can significantly affect its behavior as magnetic sensors [18]. In the characterization analysis of sensors and other detection equipment, the accurate waveforms flux is usually needed, and it is necessary to analyze the electromagnetic fields, and the hysteresis characteristics of grain-oriented electrical steel must be taken into account [1]. To study the hysteresis characteristics, firstly, the hysteresis characteristics of oriented electrical steel should be simulated, namely, the establishing of a mathematical model of hysteresis grainoriented electrical steel. In this paper, the Fe-3\% Si grainoriented electrical steel hysteresis loop model was studied, and a new model of hysteresis loop was proposed.

\section{Experimental}

Conventional grain-oriented (CGO) electrical steel sheets and high permeability grain-oriented (HGO) electrical steel sheets were the tested materials in this paper. $\mathrm{CGO}$ and $\mathrm{HGO}$ electrical steel were named $\mathrm{CC} 1$ and $\mathrm{CH} 1$, respectively, and the chemical composition is shown in Table 1.

The samples were manufactured as standard Epstein samples, and the sample size is $350 \mathrm{~mm} \times 30 \mathrm{~mm} \times 0.3 \mathrm{~mm}$. Initial magnetic properties of $\mathrm{CCl}$ and $\mathrm{CH} 1$ at $1.0 \mathrm{~T}$ are shown in Table 2.

A standard Epstein strip for testing is placed between the yokes, and a feedback control system implemented in LabVIEW was used to control the flux density to have repeatable and comparable measurements. The whole measurement was conducted in a magnetic shielding room and the computer was put in another room to avoid interference [2-5].

\section{Formula Derivation and Modeling}

Since the hysteresis loop is nearly symmetrical concerning the loop curve origin point, according to the Fourier series, the magnetic induction intensity and magnetic field strength could be expanded, respectively. Symmetry of the origin makes Fourier series contain only odd entries, and the expanded equations are as shown in

$$
\begin{aligned}
H= & H_{1} \sin \left(\omega t+\varphi_{1}\right)+H_{3} \sin \left(\omega t+\varphi_{3}\right) \\
& +H_{5} \sin \left(\omega t+\varphi_{5}\right)+\cdots \\
B= & B_{1} \sin \left(\omega t+\psi_{1}\right)+B_{3} \sin \left(\omega t+\psi_{3}\right) \\
& +B_{5} \sin \left(\omega t+\psi_{5}\right)+\cdots .
\end{aligned}
$$

To simplify the calculation, magnetic induction intensity is taken as the first term, and the magnetic field strength is viewed as a function of magnetic induction intensity, and magnetic field strength should contain unlimited expansion terms. In the derivation and calculation of the model, only the first two terms were used, and the basic model of the hysteresis loop could be obtained. Hence,

$$
\begin{aligned}
& B=B_{1} \sin (\omega t) \\
& H=B_{1}\left(\frac{1}{\mu_{1}} \sin \left(\omega t+\varphi_{1}\right)+\frac{1}{\mu_{3}} \sin \left(3 \omega t+\varphi_{3}\right)\right) .
\end{aligned}
$$

Equation (3) reflects the nonlinear relationship between the magnetic field strength and magnetic induction intensity, in which $\varphi$ represents the impact hysteresis. Values of $\varphi_{1}, \varphi_{3}, \mu_{1}$, and $\mu_{3}$ are influenced by magnetic field strength, which are determined by hysteresis loop. Once values of $\varphi_{1}, \varphi_{3}, \mu_{1}$, and $\mu_{3}$ are determined, ideal hysteresis loop could be simulated. If the hysteresis loop is smooth, the fitting degree will be high.

Undetermined parameters could be determined by the fixed angle method. In (2) and (3), $H_{1}$ represents magnetic field strength $H$ amplitude in the hysteresis loop. Making $\omega t$ take different angles, respectively, in this paper, we chose 0 , $\pi / 3, \pi / 2$, and $2 \pi / 3$; the corresponding $H_{1}, H_{2}, H_{3}$, and $H_{4}$ could be found; taking $\mathrm{H}_{4}$ back to the equation of magnetic field strength, the following equations could be obtained:

$$
\begin{aligned}
H_{1} & =B_{1}\left(\frac{1}{\mu_{1}} \sin \varphi_{1}+\frac{1}{\mu_{3}} \sin \varphi_{3}\right), \\
H_{2} & =B_{1}\left(\frac{1}{\mu_{1}} \sin \left(\frac{\pi}{3}+\varphi_{1}\right)+\frac{1}{\mu_{3}} \sin \left(\pi+\varphi_{3}\right)\right) \\
& =B_{1}\left(\frac{1}{2 \mu_{1}} \sin \varphi_{1}+\frac{\sqrt{3}}{2 \mu_{1}} \cos \varphi_{1}-\frac{1}{\mu_{3}} \cos \varphi_{3}\right), \\
H_{3} & =B_{1}\left(\frac{1}{\mu_{1}} \sin \left(\frac{\pi}{2}+\varphi_{1}\right)+\frac{1}{\mu_{3}} \sin \left(\frac{3 \pi}{2}+\varphi_{3}\right)\right) \\
& =B_{1}\left(\frac{1}{\mu_{1}} \cos \varphi_{1}-\frac{1}{\mu_{3}} \cos \varphi_{3}\right), \\
H_{4} & =B_{1}\left(\frac{1}{\mu_{1}} \sin \left(\frac{2 \pi}{3}+\varphi_{1}\right)+\frac{1}{\mu_{3}} \sin \left(2 \pi+\varphi_{3}\right)\right) \\
& =B_{1}\left(\frac{\sqrt{3}}{2 \mu_{1}} \cos \varphi_{1}-\frac{1}{2 \mu_{1}} \sin \varphi_{1}+\frac{1}{\mu_{3}} \sin \varphi_{3}\right) .
\end{aligned}
$$

Adding $\mathrm{H}_{2}$ and $\mathrm{H}_{4}$, the following calculation could be obtained:

$$
H_{2}+H_{4}=B_{1} \frac{\sqrt{3}}{\mu_{1}} \cos \varphi_{1}
$$

After transformation, $\varphi_{3}$ could be deleted; hence,

$$
H_{1}+H_{2}=B_{1}\left(\frac{3}{2 \mu_{1}} \sin \varphi_{1}+\frac{\sqrt{3}}{2 \mu_{1}} \cos \varphi_{1}\right) \text {. }
$$


TABLE 2: Initial magnetic properties of $\mathrm{CCl}$ and $\mathrm{CH} 1$ steel at $1.0 \mathrm{~T}$.

\begin{tabular}{lcccc}
\hline Sample & Iron loss $(\mathrm{W} / \mathrm{kg})$ & Coercive force $(\mathrm{A} / \mathrm{m})$ & Relative permeability & MBNrms $(\mathrm{mV})$ \\
\hline $\mathrm{CC} 1$ & 0.711 & 282.9 & 799.4 & 0.391 \\
$\mathrm{CH} 1$ & 0.668 & 287.6 & 2473.7 & 0.447 \\
\hline
\end{tabular}

To delete $\mu_{1}$, sine expression concerning $\varphi_{1}$ could be obtained through calculation; hence,

$$
2 H_{1}+H_{2}-H_{4}=B_{1}\left(\frac{3}{\mu_{1}} \sin \varphi_{1}\right) .
$$

Delete $\mu_{1}$, and the expression concerning $\varphi_{1}$ could be obtained as

$$
\begin{aligned}
\frac{2 H_{1}+H_{2}-H_{4}}{H_{2}+H_{4}} & =\sqrt{3} \tan \varphi_{1}, \\
\varphi_{1} & =\arctan \left(\frac{2 H_{1}+H_{2}-H_{4}}{\sqrt{3}\left(H_{2}+H_{4}\right)}\right) .
\end{aligned}
$$

Value of $\mu_{1}$ could be calculated as

$$
\mu_{1}=\frac{3 B_{1}}{2 H_{1}+H_{2}-H_{4}} \sin \varphi_{1}
$$

or

$$
\mu_{1}=\frac{\sqrt{3} B_{1}}{H_{2}+H_{4}} \cos \varphi_{1} .
$$

Through a similar method, sine and cosine expression concerning $\varphi_{3}$ could be calculated as

$$
\begin{gathered}
H_{1}-H_{2}+H_{4}=3 \frac{B_{1}}{\mu_{3}} \sin \varphi_{3}, \\
H_{2}+H_{4}-\sqrt{3} H_{3}=\sqrt{3} \frac{B_{1}}{\mu_{3}} \cos \varphi_{3} .
\end{gathered}
$$

To calculate $\varphi_{3}$, it is necessary to delete $\mu_{3}$ first, and the following transformation could be made:

$$
\tan \varphi_{3}=\frac{H_{1}-H_{2}+H_{4}}{\sqrt{3}\left(H_{2}+H_{4}\right)-3 H_{3}} .
$$

The value of $\varphi_{3}$ could be calculated as

$$
\varphi_{3}=\arctan \left(\frac{H_{1}-H_{2}+H_{4}}{\sqrt{3}\left(H_{2}+H_{4}\right)-3 H_{3}}\right) .
$$

$\mu_{3}$ could be calculated when taking it back; hence,

$$
\mu_{3}=\frac{3 B_{1} \sin \varphi_{3}}{H_{1}-H_{2}+H_{4}}
$$

or

$$
\mu_{3}=\frac{3 B_{1} \cos \varphi_{3}}{\sqrt{3}\left(H_{2}+H_{4}\right)-3 H_{3}} .
$$

After the hysteresis loop was obtained after experiment, other hysteresis loop parameters could be determined by linear interpolation.

For given $B_{k}\left(B_{j}<B_{k}<B_{j+1}\right)$, linear interpolation could be applied to calculate model parameters $\varphi_{1}, \varphi_{3}, \mu_{1}$, and $\mu_{3}$.

If $\varphi_{j}, \varphi_{j+1}, B_{j}, B_{k}$, and $B_{j+1}$ are given, calculations could be made as follows:

$$
\varphi_{k}=\varphi_{j}+\frac{\varphi_{j+1}-\varphi_{j}}{B_{j+1}-B_{j}}\left(B_{k}-B_{j}\right) .
$$

When $k=1,3, \varphi_{1}$ and $\varphi_{3}$ could be calculated, respectively, as

$$
\begin{aligned}
B & =B_{1} \sin \left(\omega t+\varphi_{1}\right)+B_{3} \sin \left(\omega t+\varphi_{3}\right) \\
& =H_{1}\left(\mu_{1} \sin \left(\omega t+\varphi_{1}\right)+\mu_{3} \sin \left(3 \omega t+\varphi_{3}\right)\right) .
\end{aligned}
$$

$\mu_{1} H_{k}$ and $\mu_{3} H_{k}$ could be calculated by linear interpolation.

If $\mu_{1 j}, \mu_{1 j+1}, H_{1 j}, H_{1 j+1}$, and $H_{k}$ were given, the following calculations could be made:

$$
\begin{aligned}
L_{1}(x)= & \frac{x_{k+1}-x}{x_{k+1}-x_{k}} y_{k}+\frac{x-x_{k}}{x_{k+1}-x_{k}} y_{k+1}, \\
\mu_{1} H_{k}= & \frac{H_{1 j+1}-H_{k}}{H_{1 j+1}-H_{1 j}} \cdot \mu_{1 j} H_{1 j}+\frac{H_{k}-H_{1 j}}{H_{1 j+1}-H_{1 j}} \\
& \cdot \mu_{1 j+1} H_{1 j+1} .
\end{aligned}
$$

The two-point linear interpolation could be expressed as

$\mu_{1}$

$$
=\frac{\mu_{1 j}\left(H_{1 j+1}-H_{1 j}\right) H_{1 j}+\mu_{1 j+1}\left(H_{k}-H_{1 j}\right) H_{1 j+1}}{\left(H_{1 j+1}-H_{1 j}\right) H_{k}} .
$$

Similarly, if $\mu_{1 j}, \mu_{1 j+1}, H_{1 j}, H_{1 j+1}$, and $H_{k}$ were given, $\mu_{3}$ could be calculated as

$$
=\frac{\mu_{3 j}\left(H_{3 j+1}-H_{3 j}\right) H_{3 j}+\mu_{3 j+1}\left(H_{k}-H_{3 j}\right) H_{3 j+1}}{\left(H_{3 j+1}-H_{3 j}\right) H_{k}} .
$$

New models of hysteresis loop were derived above, and the calculation of undetermined coefficient method was given utilizing interpolation step, and the model is simple and convenient.

Least squares curve fitting method is a common fitting method. When using such method to make calculation of undetermined coefficients, since the basic relationship equations of the magnetic induction density and magnetic 


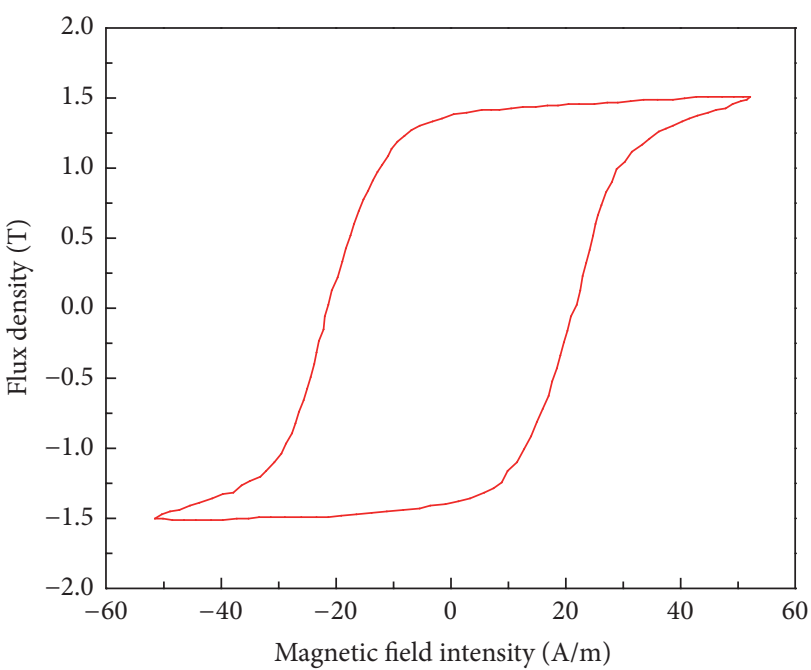

(a)

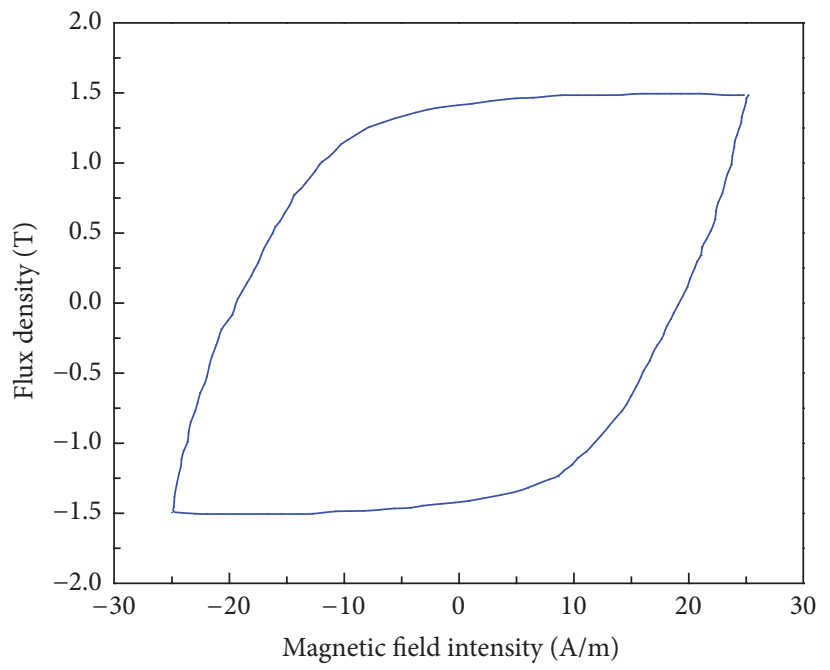

(c)

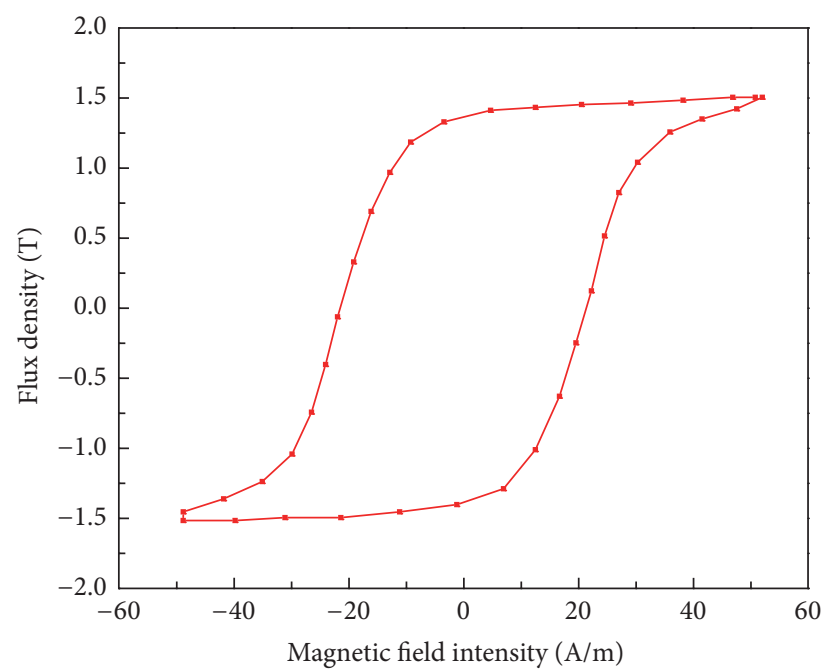

(b)

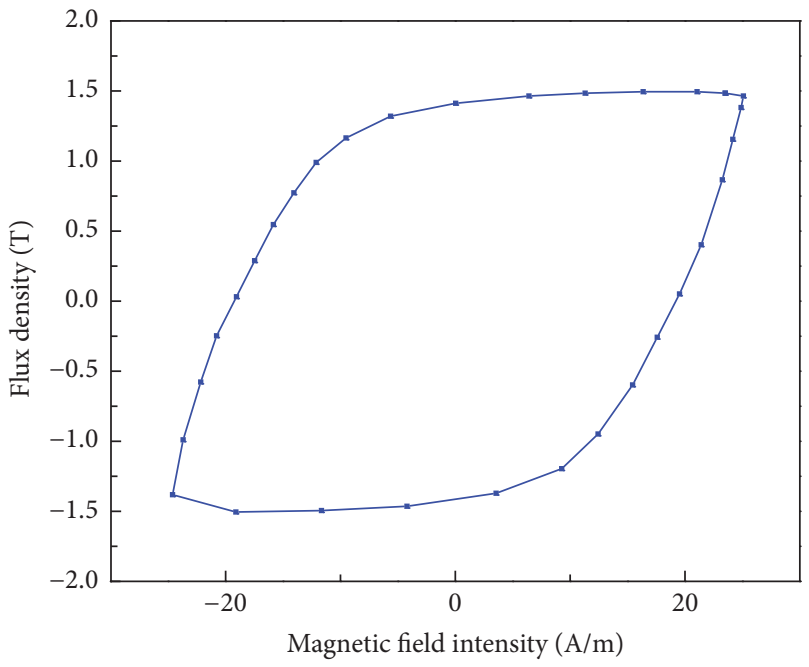

(d)

Figure 1: Comparison of original and fitting B-H loop graph of (a, b) $\mathrm{CC1}$ and (c, d) CH1 grain-oriented electrical steel.

field strength have been given, undetermined parameters of the model can be determined by magnetic field strength and magnetic induction density values of a set of experimental measurements. Assuming $\left(H_{1}, B_{1}\right),\left(H_{2}, B_{2}\right),\left(H_{3}, B_{3}\right), \ldots$ are known and $H$ is the measured magnetic induction intensity and $H^{\prime}$ is the calculated magnetic induction intensity, then undetermined coefficients $\varphi_{1}, \varphi_{3}, \mu_{1}$, and $\mu_{3}$ could be determined by residual square difference minimization of the measured and calculated values. Hence,

$$
Q=\sum_{k=1}^{n} e_{k}^{2}=\sum_{k=1}^{n}\left[B_{k}-f\left(B_{k}, \mu_{1}, \mu_{3}, \psi_{1}, \psi_{3}\right)\right]^{2} .
$$

Undetermined coefficients of $B-H$ loop model can be calculated while $Q$ takes the minimum value in (21). Hence,

$$
\begin{aligned}
& \frac{\partial Q}{\partial \mu_{1}}=0, \\
& \frac{\partial Q}{\partial \mu_{3}}=0,
\end{aligned}
$$

$$
\begin{aligned}
\frac{\partial Q}{\partial \psi_{1}} & =0, \\
\frac{\partial Q}{\partial \psi_{3}} & =0 .
\end{aligned}
$$

The equations consist of the above four equations; the undetermined coefficients could be obtained, which could be back to the original hysteresis models. The relationship between the magnetic induction intensity and magnetic field strength can be obtained, illustrating the hysteresis loop model.

\section{Model Application}

The proposed model was applied for $B-H$ curve fitting, as shown in Figure 1. The error of the single data point is controlled within $5 \%$, achieving fine fitting effect. 
Figure 1 simulation results show fine fitting result, and it is suitable for the working condition of silicon steel applied in the cigarette sensor machines, which is the possible complex magnetization condition in normal working conditions.

\section{Conclusions}

Expansion of the magnetic induction intensity and magnetic field strength was made according to the Fourier series, respectively. The magnetic field strength was determined by the magnetic induction intensity, and the undetermined parameters of angles, magnetic induction intensity, and the magnetic field strength could be determined by fixed angle method and the least squares method. General application steps of the model were given. The proposed model was applied for $B-H$ curve fitting, and the error of the single data point is controlled within $5 \%$, achieving fine fitting effect.

\section{Competing Interests}

No potential conflict of interests was reported by the authors.

\section{Authors' Contributions}

Hao Wang, Jianbo Zhan, and Zhenhua Yu contributed equally to the paper.

\section{Acknowledgments}

The financial support from the National Natural Science Foundation of China (Grant no. 51404159), Science and Technology Project of Hebei Province (Grant no. 152177180), and Science and Technology Project of Qinhuangdao City (Grant no. 201502A037) is greatly acknowledged.

\section{References}

[1] S. Boukhtache, B. Azoui, and M. Féliachi, "Optimized model for magnetic hysteresis in silicon-iron sheets by using the simulated annealing algorithm," International Journal of Applied Electromagnetics and Mechanics, vol. 30, no. 1-2, pp. 1-7, 2009.

[2] H. Wang, C. S. Li, T. Zhu, N. Chukwuchekwa, B. Cai, and G. Huo, "Effect of ball scribing on relative permeability of grain-oriented electrical steel," Acta Metallurgica Sinica (English Letters), vol. 26, no. 5, pp. 618-622, 2013.

[3] H. Wang, C.-S. Li, and T. Zhu, "Hard magnetization direction and its relation with magnetic permeability of highly grainoriented electrical steel," International Journal of Minerals, Metallurgy and Materials, vol. 21, no. 11, pp. 1077-1082, 2014.

[4] H. Wang, C.-S. Li, G. Huo, B. Cai, and N. Mohamed, "Effect of ball scribing on iron loss of CGO and HGO electrical steel," Journal of Harbin Institute of Technology (New Series), vol. 20, no. 3, pp. 99-103, 2013.

[5] H. Wang, C.-S. Li, R.-B. Mei, and G. Huo, "Effect of ball scribing on magnetic shielding efficiency of grain-oriented silicon steel," Journal of Iron and Steel Research, International, vol. 21, no. 7, pp. 679-684, 2014.

[6] N. H. Vrijsen, J. W. Jansen, and E. A. Lomonova, "Prediction of magnetic hysteresis in the force of a prebiased E-core reluctance actuator," IEEE Transactions on Industry Applications, vol. 50, no. 4, pp. 2476-2484, 2014.

[7] T. J. Fal, M. L. Plumer, J. P. Whitehead, J. I. Mercer, J. Van Ek, and K. Srinivasan, "Simulations of magnetic hysteresis loops for dual layer recording media," Applied Physics Letters, vol. 102, no. 20, Article ID 202404, 2013.

[8] A. D. Theocharis, J. Milias-Argitis, and T. Zacharias, "Singlephase transformer model including magnetic hysteresis and eddy currents," Electrical Engineering, vol. 90, no. 3, pp. 229-241, 2008.

[9] A. D. Theocharis, J. Milias-Argitis, and T. Zacharias, “Threephase transformer model including magnetic hysteresis and eddy currents effects," IEEE Transactions on Power Delivery, vol. 24, no. 3, pp. 1284-1294, 2009.

[10] N. C. Pop and O. F. Caltun, "Jiles-Atherton magnetic hysteresis parameters identification," Acta Physica Polonica A, vol. 120, no. 3, pp. 491-496, 2011.

[11] H. Tanaka, K. Nakamura, and O. Ichinokura, "Magnetic circuit model considering magnetic hysteresis," IEEJ Transactions on Fundamentals and Materials, vol. 134, no. 4, pp. 243-249, 2014.

[12] Z. Cheng, N. Takahashi, B. Forghani et al., "Analysis and measurements of iron Loss and FLUX inside silicon steel laminations," IEEE Transactions on Magnetics, vol. 45, no. 3, pp. 1222-1225, 2009.

[13] E. Cardelli, A. Faba, G. Finocchio, and B. Azzerboni, "Mathematical modelling of magnetic hysteresis in exchange-bias spin valves," IEEE Transactions on Magnetics, vol. 48, no. 11, pp. 33673370, 2012.

[14] H. Y. Lu, J. G. Zhu, and S. Y. R. Hui, "Measurement and modeling of thermal effects on magnetic hysteresis of soft ferrites," IEEE Transactions on Magnetics, vol. 43, no. 11, pp. 3952-3960, 2007.

[15] W. Williams and D. J. Dunlop, "Simulation of magnetic hysteresis in pseudo-single-domain grains of magnetite," Journal of Geophysical Research, vol. 100, no. 3, pp. 3859-3871, 1995.

[16] H. Mamiya and B. Jeyadevan, "Magnetic hysteresis loop in a superparamagnetic state," IEEE Transactions on Magnetics, vol. 50, no. 1, Article ID 4001604, 2014.

[17] H. Y. Lu, J. G. Zhu, and S. Y. R. Hui, "Measurement and modeling of thermal effects on magnetic hysteresis of soft ferrites," IEEE Transactions on Magnetics, vol. 43, no. 11, pp. 3952-3960, 2007.

[18] J. An and D.-S. Kwon, "Modeling of a magnetorheological actuator including magnetic hysteresis," Journal of Intelligent Material Systems and Structures, vol. 14, no. 9, pp. 541-550, 2003. 

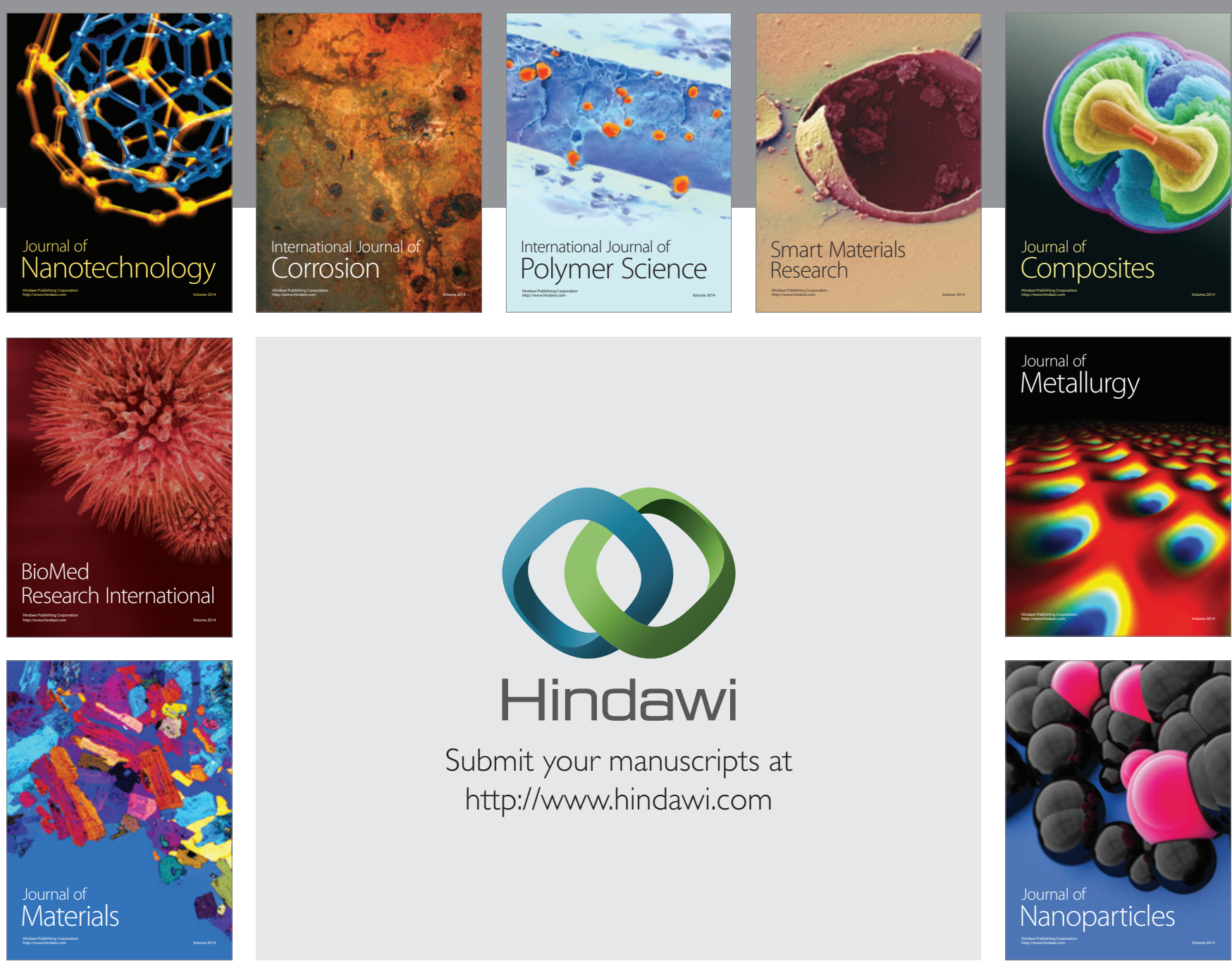

\section{Hindawi}

Submit your manuscripts at

http://www.hindawi.com

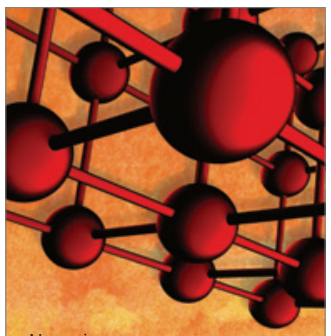

Materials Science and Engineering
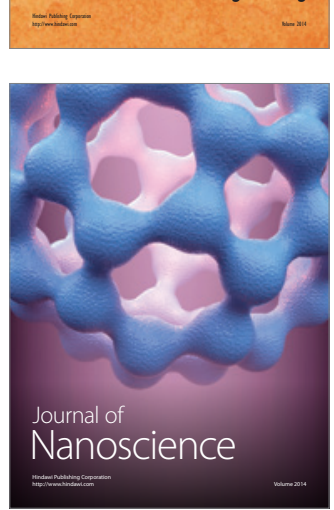
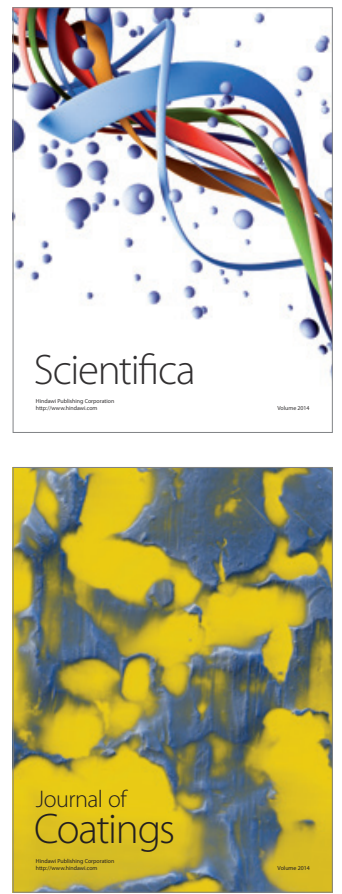
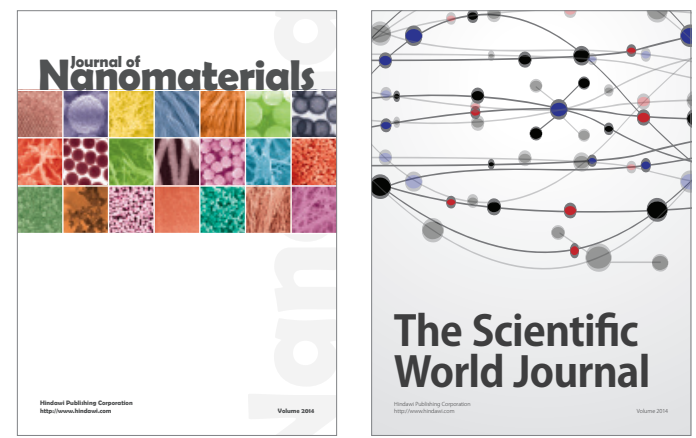

The Scientific World Journal
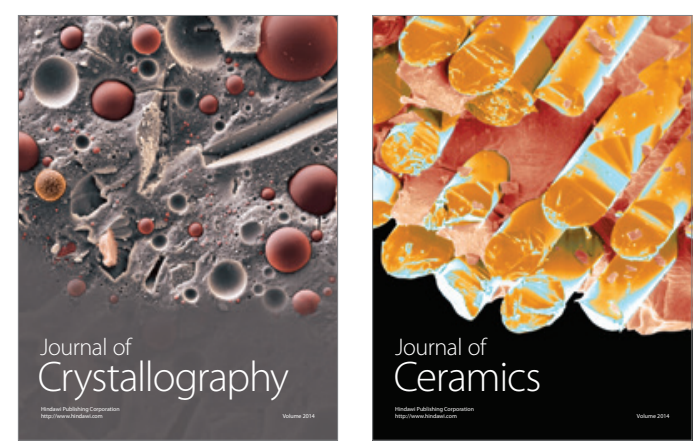
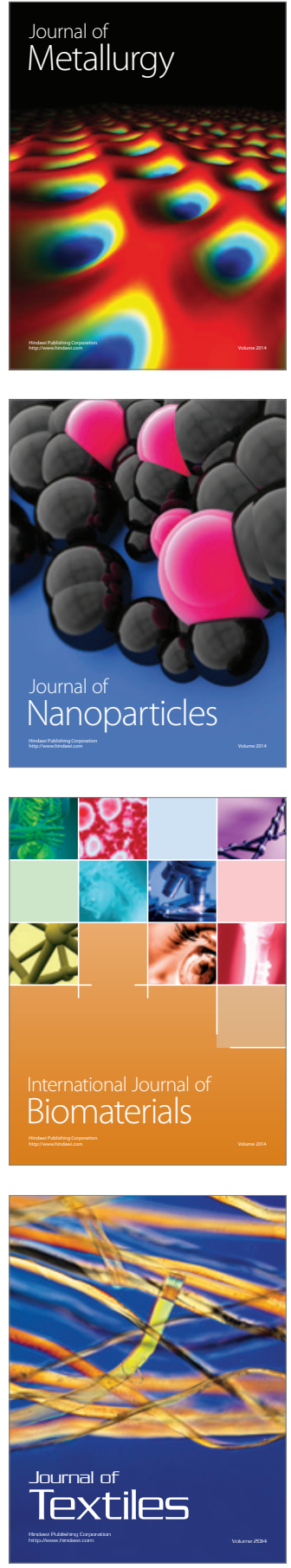